\title{
Tendência, ciclos e sazonalidade nos preços spot do café brasileiro na NYBOT
}

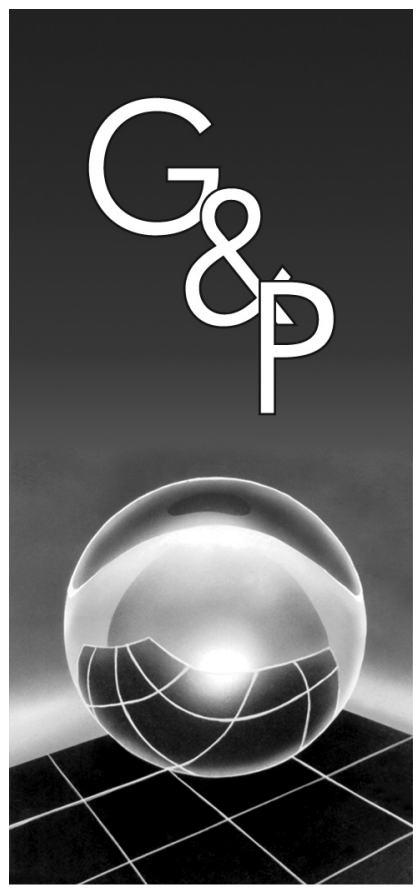

Wagner Moura Lamounier
Resumo

Pretendeu-se com este trabalho de pesquisa detectar a existência dos componentes estocásticos elou determinísticos de tendência, ciclo, e sazonalidade nos preços do mercado spot da principal commodity agrícola do Brasil: o café. A metodologia empregada referiu-se a análises no domínio do tempo (para a análise de tendência e sazonalidade) e análise no domínio da freqüência, também conhecida como análise espectral, para o estudo da presença de ciclos de preços. Os resultados encontrados mostram que a tendência existente nos preços do café se configurou como uma composição de tendências dos tipos determinística e estocástica. Com relação aos ciclos existentes nos preços do café, tem-se que a análise espectral para os dados conjuntos de toda a amostra (janeiro de 1946 a dezembro de 2000) confirmou a incidência de um ciclo de média duração, existente no intervalo de 22 a 44 meses. Em relação à análise da sazonalidade nos preços, observou-se que a mesma, enquanto um componente de influência no comportamento dos preços do café no mercado internacional, não é da forma determinística e regular, pelo contrário, é de natureza estocástica, variando em função do período do tempo.

Palavras-chave: Tendência. Ciclos. Sazonalidade. Preços do café.

\section{Introdução}

A capacidade de intervenção estatal na economia vem se esgotando nos anos recentes. O déficit público cresceu significativamente no período pós Plano Real, e ao que se refere às políticas agrícolas de controle de preços e estoques, e de financiamento da produção agropecuária, observa-se um gradativo redirecionamento para instrumentos de mercado como fontes de financiamento da produção, assim como para o controle de riscos nas atividades rurais.

Nesse sentido, o controle do risco, via conhecimento das especificidades dos mercados em que atua, tem se configurado cada vez mais como um dos principais aspectos na elevação da eficiência das diversas atividades econômicas. Todavia, o que se pode observar é que essas possibilidades de ganho, advindas de uma gestão mais eficiente dos riscos, ainda não são plenamente aproveitadas por grande parte dos agentes econômicos envolvidos nesses mercados, em razão da imperfeita compreensão acerca da natureza das flutuações dos preços, que se não podem ser evitadas, ao menos deveriam ser melhor compreendidas pelos agentes econômicos ligados a esses mercados, a fim de otimizar suas decisões de hedge e de especulação.
Particularmente no que tange às séries históricas de preços, tem-se que a análise do comportamento destas apresenta papel fundamental dentro da economia, visto que praticamente todas as fases das relações econômicas estão diretamente relacionadas aos preços. A análise dos preços agropecuários se configura como ponto de fundamental interesse, dada a ampla gama de inter-relações com outros setores de atividade e agentes econômicos envolvidos com esse setor, principalmente em uma economia com vocação agropecuária como a do Brasil, em que as atividades ligadas ao agronegócio são responsáveis por aproximadamente $32 \%$ do PIB, conforme aponta Rodrigues (2001).

Apesar da pouca liquidez da maioria dos contratos futuros de commodities agropecuárias na $\mathrm{BM} \& \mathrm{~F}$, o café se destaca como a principal commodity transacionada no mercado de futuros do Brasil. Isso se dá tanto em termos do número de contratos negociados, como em termos de volume financeiro envolvido nessas transações. Esse fato, em grande parte, é reflexo das acentuadas flutuações de preços ao longo do tempo, que caracterizam o mercado spot de café. Essas flutuações implicam que a rentabilidade nessa atividade deverá oscilar tanto para os 
cafeicultores diretamente envolvidos com a produção, quanto para os demais agentes que lidam direta ou indiretamente com essa commodity.

No que tange aos produtores de café, o conhecimento do comportamento dos preços poderá ser extremamente útil nas suas tomadas de decisões com relação ao planejamento da produção, à manutenção e formação de estoques, dentre outras, podendo assim aproveitar de maneira mais eficiente as fases de baixa e de alta nos preços, para a maximização dos lucros.

\section{Objetivos}

Em termos gerais, pretendeu-se nesta pesquisa verificar a existência dos componentes estocásticos e/ou determinísticos de tendência, ciclo, e sazonalidade na série temporal de preços do mercado spot do café, na Bolsa de Nova York, no período de janeiro de 1946 a dezembro de 2000.

Especificamente, pretendeu-se:

a) testar a validade da hipótese que postula que os preços dos produtos agrícolas em geral, possuem tendência declinante ao longo do tempo, confrontando esta hipótese com a de passeio aleatório (teoria dos mercados eficientes) para os preços do café;

b) verificar se os componentes de tendência e de sazonalidade nas séries, caso existam, são do tipo determinístico ou do tipo estocástico, discutindo as suas diferentes implicações para os agentes econômicos envolvidos;

c) determinar quais ciclos (em diferentes freqüências) têm afetado de forma mais significativa o comportamento dos preços, identificando as suas respectivas amplitudes e verificando se a periodicidade desses ciclos tem sofrido variações ao longo do tempo; e

d) verificar a incidência de variações sazonais nos preços e a possibilidade do efeito sazonal ter variado com o passar dos anos, estabelecendo por fim índices sazonais para os preços e obtendo uma série desazonalizada.

\section{Metodologia}

Uma das principais formas de análise de séries temporais se dá pela busca da decomposição das mesmas em termos de seus componentes principais. Esquematicamente, esses componentes da variação em uma série temporal podem ser caracterizados como tendências, sazonalidades e ciclos. Essa abordagem tem em Harvey $(1981,1989)$ um dos seus principais expoentes e tornouse mais conhecida no meio acadêmico como abordagem estrutural para a modelagem e análise de séries temporais.
A tendência em uma série temporal está ligada, conforme aponta Chatfield (1996), à uma mudança no nível médio da série a longo-prazo. Ou seja, a tendência irá refletir o declínio, a elevação ou a estabilidade (quando de fato não houver tendência) do valor médio da série temporal a longo-prazo; que se pode convencionar como sendo referente a observações relativas a períodos de tempo de 25 anos, aproximadamente.

Os Ciclos em uma série de tempo se referem aos movimentos recorrentes de elevação e queda em torno do nível médio da tendência e, portanto, também são relativos a dados que refletem o longo-prazo da variável em questão. De acordo com Souza (1989), deve-se ressaltar que os movimentos para cima e para baixo em torno da tendência podem ser estritamente periódicos ou aproximadamente periódicos, assim sendo, eles podem variar em comprimento e magnitude.

O terceiro componente que pode estar presente em uma série temporal é aquele relativo à Sazonalidade. Assim como o componente cíclico, a sazonalidade também está relacionada aos movimentos para cima e para baixo em torno de um valor médio, contudo, ela difere basicamente em dois aspectos do primeiro: a sazonalidade possui um comprimento constante de 12 meses, repetindo-se nesta base periódica regular e as variações sazonais podem ser observadas tendo-se por base períodos menores de tempo (médio e curto-prazo), assim sendo, os dados a serem analisados não deverão estar distribuídos em observações anuais, mas sim mensais ou trimestrais, para que seja possível a modelagem da sazonalidade.

\subsection{Análise do componente de tendência}

O primeiro passo na análise da tendência é dado verificando-se a estacionariedade da série em questão. Em termos práticos, a estacionariedade que é testada se refere à estacionariedade fraca. Isto é, deve-se verificar se a série temporal $(Y)$ terá média e variância finitas. Caso isto se confirme, a mesma será considerada estacionária em covariância. Os principais testes para verificação da estacionariedade ou não da série (chamados de testes de raízes unitárias) que serão empregados são os testes de Dickey-Fuller Expandido (ADF) e o teste de PhillipsPerron (PP).

A tendência em uma série pode ser provocada por dois tipos de fenômenos que apresentam características particulares. De acordo com a sua natureza e padrão comportamental, a tendência poderá ser caracterizada como sendo do tipo determinística ou do tipo estocástica.

A característica essencial de uma tendência determinística se refere ao fato de que a variação no nível médio de uma dada variável se dará, de forma previsível, como uma função do tempo. Ou seja, conforme apontam Pindyck e Rubinfeld (1998), e Bowerman e O'connell (1979), uma 
série de tempo $Y$, poderá evoluir em montantes absolutos em cada período de tempo, de acordo com a Equação 1:

$$
Y_{t}=T_{t}+\varepsilon_{t}
$$

em que $T_{t}$ é o componente de tendência determinística do modelo, e $\varepsilon_{t}$ é um termo de erro com média zero e variância igual a $\sigma^{2}$.

$\mathrm{O}$ valor de $Y_{t}$ poderá ser previsto de acordo com a Equação 1, sendo que o componente de tendência $\left(T_{t}\right)$ poderá assumir várias formas, tais como:

$$
\begin{gathered}
T_{t}=\varphi_{0} \\
T_{t}=\varphi_{0}+\varphi_{1} t \\
T_{t}=\varphi_{0}+\varphi_{1} t+\varphi_{2} t^{2}+\ldots . .+\varphi_{k} t^{k}
\end{gathered}
$$

Nesse tipo de modelo, a média da série poderá evoluir de forma linear ou curvilínea ao longo do tempo, sendo que uma gama enorme de possibilidades poderá ser verificada, de acordo com os sinais dos coeficientes $\left(\varphi_{i}\right)$ do polinômio.

A tendência determinística de uma série também pode assumir formas mais complexas, como a do modelo de crescimento exponencial:

$$
Y_{t}=A \cdot e^{\delta t} \varepsilon_{t}
$$

que pode ser estimado após tomar-se o logaritmo de ambos os lados da Equação 5, que resulta:

$$
\ln Y_{t}=\varphi_{0}+\varphi_{1} t+u_{t}
$$

em que: $\varphi_{0}=\ln \mathrm{A}, \varphi_{1}=\delta$, que é a taxa de crescimento da série, e $u_{t}=\ln \varepsilon_{t}$.

Todavia, o problema com essa classe de modelos de análise é que nem sempre a tendência dos dados em questão é do tipo determinística. Ao contrário, ela pode estar mudando de forma aleatória ao longo do tempo e se caracterizando como um componente de tendência do tipo estocástica.

A diferença principal entre estas duas formas de tendência se refere ao fato de que a tendência estocástica implica em uma variação percentual média na série, em dado período de tempo, porém, ao contrário da determinística, em cada período a mudança provocada pela tendência em relação ao seu nível médio será um montante aleatório, ao invés de constante dado por uma determinada taxa. Ou seja, no caso da existência de uma tendência do tipo estocástica, tem-se que os coeficientes $\left(\varphi_{k}\right)$ de uma equação para a descrição da tendência, como os apresentados nos modelos anteriores, poderão não ser constantes, mas também variarem aleatoriamente ao longo do tempo.

Existem diversos modelos de séries temporais que incorporam tendências estocásticas, e que vem ganhando destaque na bibliografia recente sobre análise de séries temporais. Nessa classe de modelos, destaca-se o modelo de passeio aleatório (random walk) dado por:

$$
Y_{t}=Y_{\mathrm{t}-1}+\varepsilon_{t}
$$

ou

$$
\Delta Y_{t}=\varepsilon_{t}
$$

em que: $\varepsilon_{t}$ é um erro do tipo ruído branco, com média 0 e variância $\sigma^{2}$, e $\Delta$ é um operador de diferenças finitas de primeira ordem.

O modelo de passeio aleatório é claramente um caso especial de um processo auto-regressivo de ordem 1 [AR(1)], dado por:

$$
Y_{t}=\gamma_{0}+\gamma_{1} Y_{t-1}+\varepsilon_{t}
$$

em que $\gamma_{0}=0$ e $\gamma_{1}=1$.

A variância da série $Y_{t}$ em questão será dependente do tempo, pois os choques exógenos $\left(\varepsilon_{t}\right)$ terão efeitos cumulativos com o passar do tempo sobre a série. Assim sendo, o processo passeio aleatório será não-estacionário, pois $\mathrm{t} \rightarrow \infty \Rightarrow \operatorname{Var}\left(Y_{\mathrm{t}}\right) \rightarrow \infty$.

Um outro modelo que incorpora tendência estocástica, e que é bastante comum na literatura acerca dessa tendência, pode ser observado nos trabalhos de Enders (1995), Hamilton (1994) e Campbell, Lo e MacKinlay (1997), que é uma variação do modelo de passeio aleatório e que incorpora uma parte determinística ao modelo de análise das mudanças na série $Y_{t}$. O modelo de passeio aleatório com intercepto (random walk plus drift), que aumenta o modelo de passeio aleatório com a inclusão de um termo constante, é dado por:

$$
Y_{t}=Y_{t-1}+\gamma_{0}+\varepsilon_{t}
$$

Assim sendo, dada uma condição inicial $Y_{0}$, a solução geral para a série $Y_{t}$ será dada por:

$$
Y_{t}=Y_{0}+\gamma_{0} t+\sum_{i=1}^{t} \varepsilon_{i}
$$

Portanto, de acordo com Enders (1995), o comportamento da série $Y_{t}$ será governado por dois componentes não-estacionários, quais sejam: uma tendência linear determinística $\left(\gamma_{0} t\right)$ e uma tendência estocástica $\left(\Sigma \varepsilon_{i}\right)$. A variação determinística em cada nova observação de $Y_{t}$ será dada por $\gamma_{0}$, e após $t$ períodos de tempo será igual a $\gamma_{0} . t$; ao passo que a variação provocada pela tendência estocástica será dada pelo somatório dos diversos choques $\varepsilon_{i}$ que terão efeitos permanentes na média de $Y_{t}$.

\subsection{Análise do componente cíclico}

A análise de séries temporais pode ser implementada segundo diversas abordagens, que nem sempre são concorrentes entre si. Esse é o caso da análise no domínio da freqüência (ou Análise Espectral), que representa uma 
forma de análise de séries temporais que fornece informações complementares àquelas propiciadas pela análise no domínio do tempo, informações estas que geralmente estão ligadas ao estudo da ocorrência de ciclos nas séries.

A essência da análise espectral reside na representação de uma série temporal $\left\{Y_{t}\right\}_{t=-\infty}^{\infty}$ como uma soma ponderada de funções periódicas do tipo seno $(\omega t)$ e coseno $(\omega t)$, em que $\omega$ representa uma frequiência particular; e na determinação de quão importantes são os ciclos de diferentes freqüências (caso eles existam), para explicar a variância de $\left\{Y_{t}\right\}_{t=-\infty}^{\infty}$ e conseqüentemente, o comportamento dessa série.

A análise espectral para verificação de ciclos em uma série temporal $Y_{t}$, tem como ponto de partida um teorema conhecido como Teorema da Representação Espectral, segundo o qual qualquer processo estacionário discreto $Y_{t}$, com freqüências pertencentes ao intervalo $[0, \pi]$, pode ser representado da seguinte maneira:

$$
\begin{gathered}
Y_{t}=\mu+\int_{0}^{\pi}[\alpha(\omega) \cdot \cos (\omega t)] d \omega+ \\
\int_{0}^{\pi}[\beta(\omega) \cdot \operatorname{sen}(\omega t)] d \omega
\end{gathered}
$$

Esta é a forma mais comum para expressar a Representação Espectral do Processo $Y_{t}$. Portanto, da Equação 12 surge o conceito de que a análise espectral descreve o valor de uma variável $Y_{t}$ como uma soma ponderada de funções periódicas da forma $\cos (\omega t)$ e sen $(\omega t)$, em que $\omega$ representa uma frequiência particular no intervalo $[0, \pi]$. O intervalo de integração é $[0, \pi]$ e não $[0, \infty]$, devido à seguinte característica das funções trigonométricas do tipo cosseno: $\cos [(\omega+n \pi) t]=\{\cos \omega t \forall n$ e $t$ inteiros, com $v$ par $\}$ e $\cos [(\omega+n \pi) t]=\{\cos (\pi-\omega) t \forall$ $n$ e $t$ inteiros, com $n$ impar $\}$. Assim sendo, variações em freqüências maiores que $\pi$ não poderão ser distinguidas de variações nas freqüências correspondentes ao intervalo $[0, \pi]$, pois as mesmas irão se sobrepor.

A freqüência angular $\omega=\pi$, medida em termos de radianos por unidade de tempo, é conhecida como a Frequiência de Nyquist, e denota o valor mais alto com que se pode obter informações sobre ciclos periódicos, em um conjunto de dados. Portanto, tem-se que o ponto principal da introdução da Equação 12, se refere ao fato de que ela indica que cada freqüência no intervalo $[0, \pi]$ pode contribuir para a variação de uma série de tempo $Y_{t}$ qualquer.

Assim como na análise de uma série temporal no domínio do tempo, o conhecimento da Função de Autocovariância, dada por $\gamma(k)=E\left\{\left[Y_{t}-\mu\right]\left[Y_{t+k}-\mu\right]\right\}=C o v$ $\left[Y_{t}, Y_{t+k}\right]$ é de valor imprescindível para descrever a estrutura de tal processo. $\mathrm{Na}$ análise do domínio da frequiência existe uma função chamada Função de Densidade Espectral, ou simplesmente Espectro Populacional do processo
$\left\{Y_{t}\right\}_{t=-\infty}^{\infty}$, cuja integral definida em $[0, \pi]$ e chamada de Função de Distribuição Espectral é a representação espectral da função de autocovariância. Esse conceito também se configura em um desenvolvimento teórico de grande importância para a compreensão da estrutura variacional do processo. O Espectro Populacional do processo $\left\{Y_{t}\right\}_{t=-\infty}^{\infty}$ pode ser escrito como:

$$
f_{y}(\omega)=\frac{1}{2 \pi}\left[\gamma_{0}+2 \sum_{j=1}^{\infty} \gamma_{j} \cos (\omega j)\right]
$$

Conforme aponta Hamilton (1994), como o espectro representa uma função contínua de $\omega$, definida no conjunto dos números reais e $\gamma_{j}$ representa as autocovariâncias de um processo estacionário, tem-se que $f_{Y}(\omega)$ terá um valor positivo para qualquer $\omega$. Assim como ocorre na análise do domínio do tempo, em que a importância prática da função de autocorrelação não se dá com a interpretação direta dos seus coeficientes, mas sim com a análise do correlograma da série; na análise espectral, os coeficientes do espectro não representam o principal ponto de interesse para análise. É o gráfico do espectro, que também é chamado de espectro, que fornecerá as informações relevantes acerca da variância da série $Y_{t}$ e sobre quais as freqüências que contribuem para um comportamento cíclico dessa série.

Contudo, as séries temporais a serem analisadas empiricamente, geralmente são amostras finitas da população $\left\{Y_{t}\right\}_{t=-\infty}^{\infty}$ e, portanto, não se pode estimar, a partir delas, o número infinito de parâmetros da Equação 13. Portanto,, assim como ocorre nos modelos de análise estatística e econométrica "tradicionais"; geralmente o que se tem para a implementação da análise espectral são amostras finitas dos dados populacionais das séries de tempo a serem analisadas. Deve-se trabalhar com um conceito análogo ao do espectro populacional, para a análise espectral de dados amostrais. Este conceito é conhecido na literatura como o Periodograma Amostral, ou simplesmente Periodograma e a forma geral dos estimadores consistentes do espectro $f_{y}(\omega)$ da Equação 13, apontada por Judge et al. (1988), é dada pela seguinte expressão:

$$
\tilde{f}_{y}(\omega)=\frac{1}{2 \pi}\left(\lambda_{0} c_{0}+2 \sum_{j=1}^{M_{T}} \lambda_{j} c_{j} \cos \omega_{j}\right)
$$

em que a seqüência de pesos $\lambda_{j}=\left\{\lambda_{0}, \lambda_{1}, \lambda_{2}, \ldots \ldots . ., \lambda_{M T}\right\}$ é chamada de janela de defasagem (ou de suavização), e o termo $M_{T}(<N)$ é chamado de ponto de truncamento. Uma regra sugerida por Chatfield (1996) para a escolha do valor para o ponto de truncamento é a de que deve-se escolher um ponto de truncamento $\left(M_{T}\right)$ dado por:

$$
M_{T}=2 \cdot \sqrt{N}
$$

De acordo com o sugerido por Rausser e Cargill (1970), para se verificar se em um espectro estimado existem 
picos estatisticamente significativos, pode-se empregar dois critérios: $\mathrm{O}$ primeiro se refere à análise visual da função de densidade espectral estimada, buscando identificar se alguns picos se destacam nesse espectro em relação aos demais. O segundo critério, mais rigoroso, é baseado no teste da hipótese (nula) de que o espectro estimado não é estatisticamente diferente do espectro de um processo puramente aleatório (ruído branco). Isto é feito comparando-se os valores estimados do periodograma com uma distribuição exponencial.

\subsection{Análise do componente sazonal}

Diversos métodos podem ser empregados para a análise do componente sazonal em uma série de tempo; desde análise visual do gráfico da série, que se apresenta como a mais simples opção (porém útil), passando pelos Modelos de Regressão Linear com Variáveis Independentes Binárias (Variáveis Dummy) até a utilização de métodos mais sofisticados como a propiciada pelos Modelos de Análise Espectral (que trata a sazonalidade como um ciclo de período fixo igual a 12 meses), dos Modelos de Box e Jenkins Sazonais (SARIMA) e de Alisamento Sazonal.

Para uma série $Y_{t}$ mensal que apresente o componente sazonal, os diversos pontos amostrais deverão apresentar algum grau de correlação com os dados correspondentes com lags e leads de 12 meses. Ou seja, espera-se a existência de correlação entre $Y_{t-12}$ e $Y_{t+12}$ com os valores de $Y_{t}$. Essas correlações deverão se manifestar na função de autocorrelação amostral, dada pela Equação 16, pela presença de picos significativos para os valores estimados de $\hat{\rho} k$ em que $k=12,24,36,48$ e assim por diante.

$$
\hat{\rho}_{k}=\frac{\sum_{t=1}^{T-k}\left(Y_{t}-\bar{Y}\right)\left(Y_{t+k}-\bar{Y}\right)}{\sum_{t=1}^{T}\left(Y_{t}-\bar{Y}\right)^{2}}
$$

A mensuração dos efeitos ou impactos, que os diferentes meses do ano exercem sobre a série de tempo em questão, pode ser efetuada com a estimação de índices de sazonalidade, que de forma agregada representam o componente sazonal. Abraham e Ledolter (1983) descrevem o componente sazonal e os referidos índices, com a ajuda da Equação 17:

$$
S_{t}=\sum_{i=1}^{s} \delta_{i} I N D_{t i}
$$

em que; $I N D_{t i}=1$ se t se refere ao período sazonal $i$, e $I N D_{t i}=0$ para os demais períodos, $\delta_{i}$ representam os valores dos índices que deverão ser adicionados ou subtraídos da série sazonal a fim de se eliminar esse efeito, e $\mathrm{s}$ representa o número de períodos de sazonalidade que poderão ocorrer. Por exemplo, $s$ será igual a 12 se o fenômeno sazonal a ser analisado ocorrer mensalmente ao longo do ano, e s será igual a 4 se o fenômeno sazonal estiver ligado às estações do ano (trimestral).

De maneira geral, a implementação do cálculo de tais índices consistirá da especificação de uma equação de regressão, que será estimada por $\mathrm{MQO}$, tendo como variável dependente a série $Y$ t, livre do componente de tendência. Assim sendo, a equação a ser estimada será:

$$
Y_{t}=\beta_{0}+\sum_{i=1}^{12} \beta_{i} D_{i t}+\varepsilon_{t}
$$

em que: $D_{i t}(i=1,2,3, \ldots .12$ e $t=1,2,3, \ldots . . n)$ são variáveis dummy, assumindo o papel das variáveis IND na Equação 17 ; $\varepsilon_{t}$ é um termo de erro aleatório e $\beta_{i}$ representa o efeito sazonal no i-ésimo período.

Como pode ser observado, a Equação 18 possui intercepto. Esse modelo não poderá ser estimado com essa especificação, pois ocorrerá aí o problema econométrico de multicolinearidade perfeita. O somatório das colunas das dummies apresentará colinearidade perfeita com a coluna de números 1 que multiplicam o coeficiente de intercepto. A matriz $\left(\mathrm{X}^{\prime} \mathrm{X}\right)^{-1}$ será singular. Assim sendo, um dos valores de $\beta_{i}$ deverá ser igualado à zero $\left(\beta_{12}\right.$, por exemplo) e, portanto, a interpretação dos índices sazonais será modificada. Cada um dos índices $\beta_{i}$ (para todo $i \neq 12$ ) representará o efeito sazonal do i-ésimo mês em relação ao valor observado no $12^{\circ}$ mês. Este tipo de modelo se adequa à modelagem da sazonalidade determinística da série. Neste caso, o efeito sazonal apresenta um comportamento relativamente estável e previsível ao longo dos anos.

Contudo, conforme o discutido por Vandaele (1983), o padrão da sazonalidade na série $Y_{t}$ poderá ser do tipo estocástico. Isso ocorrerá nos casos em que o componente sazonal possuir um comportamento instável ao longo do tempo, modificando-se de forma imprevisível. Assim sendo, a estimação de um modelo como o dado pela Equação 18 não irá ter nos seus resíduos uma série $Y_{t}$ livre do fenômeno sazonal.

Alguns modelos de séries temporais, tais como o SARIMA, desenvolvido por Box e Jenkins (1976) incorporam a sazonalidade na forma estocástica em suas estruturas, para propiciar análises e previsões mais precisas dos valores futuros da série. Os modelos sazonais de Box; Jenkins (1976), também conhecidos como modelos SARIMA $(\mathrm{p}, \mathrm{d}, \mathrm{q}) \times(\mathrm{P}, \mathrm{D}, \mathrm{Q})_{\mathrm{S}}$, visam explorar a correlação serial existente entre diferentes valores de uma série temporal $Y_{t}$. A equação fundamental deste modelo é dada pela seguinte expressão:

$$
\begin{gathered}
\left(1-\varphi_{1} B-\varphi_{2} B^{2}-\ldots .-\varphi_{p} B^{p}\right)\left(1-\Phi_{1} B^{s}-\ldots .-\right. \\
\left.\Phi_{p} B^{P s}\right)(1-B)^{d}\left(1-B^{s}\right)^{D} Y_{t}=\left(1-\theta_{1} B-\ldots .-\right. \\
\left.\theta_{q} B^{q}\right)\left(1-\Theta_{1} B^{s}-\ldots .-\Theta_{Q} B^{Q s}\right) \varepsilon_{t}
\end{gathered}
$$

$\mathrm{Na}$ primeira parte desta expressão, tem-se que o primeiro termo entre parêntesis corresponde aos coefi- 
cientes de um modelo auto-regressivo - $\mathrm{AR}(\mathrm{p})$; o segundo termo corresponde aos coeficientes de um modelo autoregressivo sazonal - $\mathrm{SAR}(\mathrm{P})$; o termo $d$ corresponde à ordem de integração da série, ou seja, ao número de diferenças tomadas e $D$ corresponde ao número de eventuais diferenças sazonais tomadas.

$\mathrm{Na}$ segunda parte desta expressão, após o sinal de igualdade, tem-se que o primeiro termo entre parêntesis corresponde aos coeficientes de um modelo do tipo média móvel - MA (q) e o segundo termo corresponde aos coeficientes de um modelo de médias móveis sazonais - SMA (Q). Utilizando-se o operador de diferenças $\nabla$, este modelo pode ser reescrito, de maneira mais compacta, como:

$$
\varphi(B) \Phi\left(B^{s}\right) \nabla^{d} \nabla_{s}^{D} Y_{t}=\theta(B) \Theta\left(B^{s}\right) \varepsilon_{t}
$$

Para se identificar um modelo SARIMA $(\mathrm{p}, \mathrm{d}, \mathrm{q}) \mathrm{x}$ $(\mathrm{P}, \mathrm{D}, \mathrm{Q})_{S}$, deve-se inicialmente determinar os valores de $d$ e $D$ por meio da análise do gráfico das funções de autocorrelação e de autocorrelação parcial. Se for observado um comportamento não-estacionário, com persistência de elevados valores para os coeficientes de autocorrelação e de autocorrelação parciais, pode-se inferir a necessidade de diferenciação da série.

Da mesma forma, se valores elevados e persistentes nos lags com defasagens próximas aos múltiplos do período sazonal (próximas a 12, 24 e 36, por exemplo) pode-se inferir também a necessidade de diferenciação sazonal da série.

\subsection{Dados e softwares estatísticos utilizados}

Para a implementação do presente projeto de pesquisa foram utilizados dados mensais secundários dos preços reais do café brasileiro no mercado spot de Nova Iorque (New York Board of Trade - NYBOT), de janeiro de 1946 a dezembro de 2000, cotados em centavos de US\$ por libra-peso de 2000.

Cada libra-peso tem 453,6 g. Para transformar dólares por saca de $60 \mathrm{~kg}$ em centavos de dólar por libra-peso, deve-se dividir 60.000 gramas por 453,6 gramas e depois dividir por 100 , o que resultará no fator 1,3228. Inversamente, para transformar o preço de Nova Iorque (US\$ centavos/libra-peso) em cotações da BM\&F (US\$/saca), deve-se multiplicar o primeiro por 1,3228.

Os dados, que se encontravam em valores nominais de US\$, foram atualizados para valores de dezembro de 2000 por meio do índice de inflação americana para o consumidor (Consumer Price Index for All Urban Consumers) do U.S. Department of Labor - Bureau of Labor Statistics.

Os principais softwares que foram empregados no trabalho de pesquisa para o processamento dos dados e dos modelos de análise nos domínios do tempo e da freqüência foram: STATISTICA 5.0 da Stat Soft Inc., o
SPSS 8.0 da SPSS Inc. e o EVIEWS 3.1 da Quantitative Micro Software.

\section{Resultados e discussão}

Com relação ao componente de tendência nos preços do café no mercado spot de Nova Iorque, pode-se verificar (Figura 1) da análise, que o nível médio dos preços da commodity brasileira apresentou, no início do período analisado, comportamento de crescimento a taxas decrescentes. Esse processo foi então revertido, e o nível médio dos preços passou a apresentar na segunda metade do período um comportamento de queda a taxas crescentes, indicando que a situação do setor cafeeiro nacional pode se tornar problemática nos próximos anos, caso essa tendência não seja revertida.

Apesar da relativa recuperação dos preços em 1995 e 1997, a análise dos cinco últimos anos da amostra estudada indica agravamento da tendência de queda nos preços; portanto, os produtores de café deverão buscar se tornarem cada vez mais eficientes em suas atividades, visando operar com sistemas otimizados, em termos de custos e produtividade, visto que os preços do café estão em um processo de queda relativa nos últimos 25 anos, o que confirma, em parte, a hipótese de Schultz de tendência de queda para os preços do café.

A não-estacionariedade das séries temporais na presente pesquisa foi verificada por meio do teste de Dickey Fuller expandido (ADF). Este teste permite verificar a estacionariedade das séries, identificando a presença de não-estacionariedade pela incidência de tendências estocásticas, determinísticas, ou a junção de ambas. O ADF consiste na estimação de uma regressão da primeira diferença da série, que poderá incorporar termos de tendência (determinística) $(\tau)$. Além disso, o modelo permite que $\Delta Y_{t}$ possa ser gerado por um modelo AR de ordem superior (inclusão dos termos $\Delta Y_{t-k}$ ). A

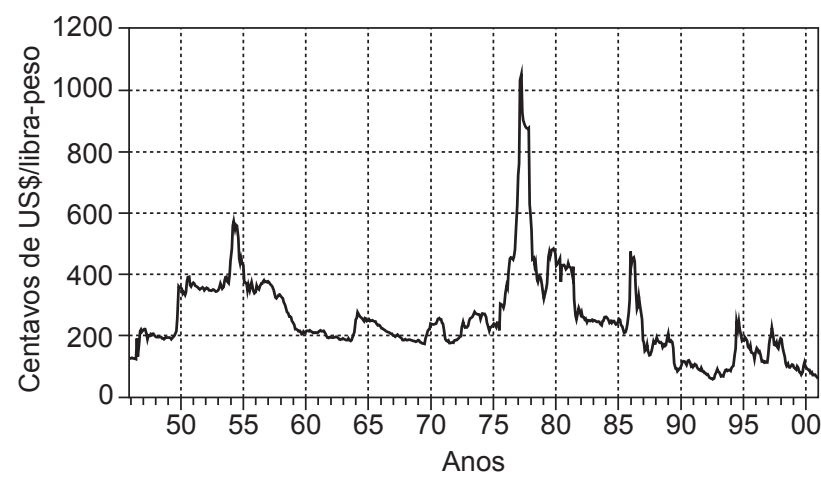

Figura 1. Preços reais do café brasileiro no mercado spot de Nova Iorque - 1946/2000 (em centavos de US\$/libra-peso de 2000). Fonte: Elaboração própria a partir de dados do USDA - Departamento de Agricultura dos Estados Unidos. 
Equação 21 representa a equação de teste do modelo ADF que foi estimado:

$$
\Delta Y_{t}=\mu+\beta \tau+(\theta-1) Y_{t-1}+\sum_{p=1}^{k-1} \phi_{p} \Delta Y_{t-p}+\varepsilon_{t}
$$

onde $\mu$ é o drift; $\tau$ é o termo de tendência linear determinística; $k$ é o número de defasagens; $\varepsilon_{t}$ é o erro aleatório do tipo white noise (ruído branco).

Os valores críticos dos testes realizados, para determinação da ocorrência ou não de raízes unitárias nos preços do café, estão sistematizados na Tabela 1. Pode-se observar, que os valores apresentados, de maneira geral, indicam que a série de preços reais do café brasileiro no mercado spot de Nova Iorque apresenta raiz unitária, isto é, ela é uma série não-estacionária em função da incidência de tendência do tipo estocástica e do tipo determinística, como se pode observar pela significância estatística dos termos $(\theta-1)$ e $\beta$, dada pelos valores de probabilidade ( $P$-Values) entre parênteses.

Assim sendo, tem-se que a série temporal dos preços do café apresenta raiz unitária e é integrada de ordem um (1). Tem-se, portanto, que choques nesses preços terão efeitos permanentes, significando que políticas agrícolas e macroeconômicas que afetem diretamente a cadeia produtiva do café terão impactos duradouros. Os resultados encontrados mostram que a tendência existente nos preços do café apresenta características determinísticas e características estocásticas.

Com relação à hipótese de ciclos existentes nos preços do café, a análise espectral para os dados conjuntos de todo o período confirmou a incidência de um ciclo de média duração existente no intervalo de 22 a 44 meses. Esses resultados indicam, que em média, poder-se-ia estabelecer um período de 36 meses de duração para este ciclo.

De acordo com o espectro estimado (Tabela 2), com uma defasagem de truncamento $M=33$ para o conjunto de observações compreendidas entre janeiro de 1946 e dezembro de 2000, os preços do café brasileiro no mercado spot de Nova Iorque apresentaram um padrão de comportamento cíclico, que se completou de três em três anos, aproximadamente.

Apesar de um tanto quanto picotado, o espectro estimado em função das frequiências (Figura 2) indica que as do intervalo dado aproximadamente por $[0,022 ; 0,045]$ são as que mais contribuem para a variância dos preços do café. Isso porque existe um pico nesse espectro, correspondente a esse intervalo de frequiências que se destaca, e que aparentemente, é significativo. Podem-se notar

Tabela 1. Resultados dos testes de raízes unitárias (Dickey-Fuller expandido). Fonte: resultados da pesquisa.

\begin{tabular}{cccccccccccc}
\hline Def. & $\mu$ & $\beta$ & $(\theta-\mathbf{1})$ & $\varphi_{1}$ & $\varphi_{2}$ & $\varphi_{3}$ & $\varphi_{4}$ & d & AIC & Sch. \\
\hline \multirow{2}{*}{1} & 8,5741 & $-0,0087$ & $-0,0228$ & 0,2172 & - & - & - & 2,0387 & 9,1895 & 9,2172 \\
& $(0,0045)$ & $(0,0918)$ & $(0,0016)$ & $(0,0000)$ & & & & & \\
2 & 9,1787 & $-0,0090$ & $-0,0248$ & 0,1997 & 0,0889 & - & - & 2,0218 & 9,1864 & 9,2206 \\
& $(0,0026)$ & $(0,0828)$ & $(0,0007)$ & $(0,0000)$ & $(0,0227)$ & & & & & \\
3 & 10,0641 & $-0,0094$ & $-0,0277$ & 0,1916 & 0,0673 & 0,1222 & - & 2,0006 & 9,1758 & 9,2168 \\
& $(0,0010)$ & $(0,0698)$ & $(0,0002)$ & $(0,0000)$ & $(0,0874)$ & $(0,0017)$ & & & \\
4 & 10,1719 & $-0,0095$ & $-0,0279$ & 0,1914 & 0,0672 & 0,1219 & 0,0023 & 1,9999 & 9,1803 & 9,2282 \\
& $(0,0010)$ & $(0,0672)$ & $(0,0002)$ & $(0,0000)$ & $(0,0885)$ & $(0,0021)$ & $(0,9530)$ & & \\
\hline
\end{tabular}

Tabela 2. Maiores valores estimados para as densidades espectrais $(M=33)$. Fonte: resultados da pesquisa.

\begin{tabular}{cccccc}
\hline Freqüiência $(\omega)$ & Período $(\mathbf{P})$ & \multicolumn{2}{c}{ Coeficientes } & Valores do periodograma & Densidades espectrais \\
\cline { 3 - 4 } & & Cosseno & Seno & & \\
\hline 0,028788 & 34,73684 & $-4,12124$ & $-2,70638$ & 8022,011 & 3613,118 \\
0,027273 & 36,66667 & 3,05165 &,- 93544 & 3361,915 & 3600,334 \\
0,030303 & 33,00000 &,- 98035 & 2,46485 & 2322,068 & 3579,865 \\
0,025758 & 38,82353 & $-3,13242$ & 3,25916 & 6743,291 & 3537,723 \\
0,031818 & 31,42857 & 1,21399 &,- 91259 & 761,171 & 3511,782 \\
0,024242 & 41,25000 & 3,35968 & $-1,32788$ & 4306,731 & 3424,561 \\
0,033333 & 30,00000 & $-1,04550$ & 2,26920 & 2059,975 & 3421,767 \\
0,034848 & 28,69565 &, 46999 &,- 54684 & 171,576 & 3319,319 \\
0,022727 & 44,00000 & 1,25145 & 3,10459 & 3697,532 & 3263,928 \\
0,036364 & 27,50000 & $-4,47815$ & 1,99434 & 7930,306 & 3210,528 \\
0,037879 & 26,40000 & 3,07311 & 1,58256 & 3943,002 & 3096,626 \\
0,021212 & 47,14286 & 1,22541 & $-3,67690$ & 4957,009 & 3059,862 \\
\hline
\end{tabular}




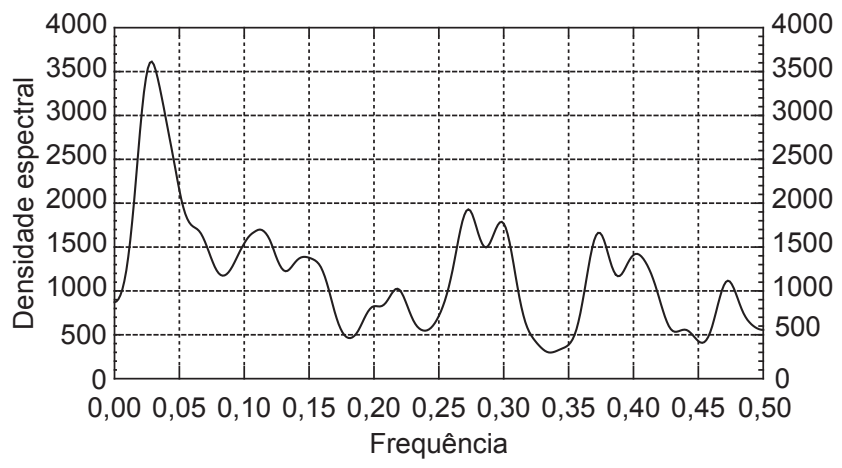

Figura 2. Espectro estimado para os preços do café de janeiro de 1946 a dezembro de 2000, com $M=33$ (em função de $\omega$ ). Fonte: Resultados da pesquisa.

também dois picos menores no intervalo de freqüências dado por $[0,25 ; 0,30]$, porém estes, aparentemente, não são de fato relevantes.

Em termos de duração, os ciclos correspondentes a essas freqüências teriam um período mínimo de aproximadamente 22 meses (correspondente à freqüência de 0,045 ) e máximo de 44 meses (definido pela frequiência de 0,022 ) para o pico mais alto; e de aproximadamente quatro meses para os picos entre as freqüências do intervalo $[0,25 ; 0,30]$. Isso indica, portanto, a existência de um ciclo de duração mais longo e um ciclo de curto prazo para esses preços.

Subdividindo a análise por décadas, a fim de verificar mudanças na periodicidade dos ciclos, constatou-se para a década de 50 a existência de um ciclo bienal, que possivelmente esteve ligado diretamente ao ciclo biológico do cafeeiro. Este ciclo biológico consiste do fato de que, de maneira geral, os cafeeiros apresentam em um ano uma grande florada, e no outro a seguir, uma florada pequena, em razão do esgotamento das plantas, que necessitam da ocorrência de um intervalo de um ano para poderem apresentar bom rendimento.

Para a década de 60, os resultados encontrados divergiram, em certa medida, dos resultados verificados para a década de 50. Neste período, o pico mais marcante e significativo ocorreu nas freqüências próximas a 0,02 , o que em termos de período, correspondeu a um ciclo de longo prazo nos preços do café de aproximadamente 60 meses (cinco anos) de duração.

Os espectros estimados para a década de 70 indicaram novamente que as frequiências próximas a 0,04 são as que mais contribuem para a variância dos preços do café. $\mathrm{O}$ maior pico verificado neste espectro se encontrou no intervalo próximo a esta frequiência, indicando que um ciclo bienal (de período igual a 24 meses) também foi marcante para a década de 70 . Pode-se notar também um pico menor nas freqüências próximas a 0,3 , que correspondeu a um período de aproximadamente quatro meses.
Os resultados encontrados para o espectro estimado dos preços do café, nos anos 80 apresentaram duas características marcantes. Primeiramente, pode-se observar que o ciclo de 24 meses foi o mais marcante em todo o conjunto de densidades espectrais estimadas, indicando que o ciclo bienal se configurou como um importante determinante do comportamento dos preços do café até o final da década de 80 , pois foi um ciclo que prevaleceu ao longo das quatro primeiras décadas analisadas. Em segundo lugar, tornou-se evidente que o ciclo sazonal, de freqüências próximas a 0,07 , novamente foi significativo nesta década, ao contrário do ocorrido na década de 70 .

Finalmente, para a década de 90 pode-se observar uma mudança no comportamento dos preços, em termos do componente de ciclos. $\mathrm{O}$ espectro estimado em função das frequiências indica que aquelas pertencentes ao intervalo dado por $[0,07 ; 0,10]$ são as que mais contribuíram para a variância dos preços do café, visto que existe um pico neste espectro que se destaca em relação aos demais. Essas frequiências correspondem ao período de 12 meses, o que configura o ciclo sazonal como o mais marcante ciclo nos preços para a década de 90 .

Os resultados encontrados na análise de ciclos nos preços do café apontam para a conclusão de que os ciclos nos preços ocorreram de forma clara nas décadas anteriores à de 90 , mas que não afetaram de maneira tão expressiva o setor após esse período. Isso porque na década de 90 , os ciclos (em sentido estrito) de fato inexistiram; apenas a sazonalidade anual dos preços foi detectada. Assim, ao persistir esse comportamento, para os anos vindouros os agentes envolvidos com esta commodity não têm razões para esperar flutuações nos preços e na rentabilidade de suas atividades em função do componente cíclico.

Em relação à análise da sazonalidade nos preços, observou-se que, para as décadas tomadas individualmente, os resultados não diferiram significativamente dos verificados para todo o período (Tabela 3 ). Constatou-se, que a sazonalidade enquanto componente de influência no comportamento dos preços do café no mercado internacional, não é da forma determinística e regular. Pelo contrário, ela é de natureza estocástica, conforme também indicaram alguns dos resultados da análise espectral apresentados anteriormente. Esse resultado se justifica em parte pela formação de estoques, tanto no mercado interno quanto no externo, que são liberados nos períodos de entressafra e que impedem oscilações mais acentuadas nos preços, bem como a caracterização de um padrão sazonal regular para estes.

Os principais resultados encontrados para as equações de sazonalidade para todo o período amostral (de 1946 a 2000) e para as décadas de 50,60, 70, 80 e 90, tomadas individualmente, podem ser observados na Tabela 3 . Como pode ser verificado, os resultados estimados apre- 
Tabela 3. Resultados dos testes de sazonalidade determinística mensal. Fonte: Resultados da pesquisa.

\begin{tabular}{|c|c|c|c|c|c|c|c|c|c|c|c|c|}
\hline \multirow[t]{2}{*}{ Período/variável } & \multicolumn{2}{|c|}{1946 a 2000} & \multicolumn{2}{|c|}{ Década de 50} & \multicolumn{2}{|c|}{ Década de 60} & \multicolumn{2}{|c|}{ Década de 70} & \multicolumn{2}{|c|}{ Década de 80} & \multicolumn{2}{|c|}{ Década de 90} \\
\hline & Ín. saz. & Prob. & Ín. saz. & Prob. & Ín. saz. & Prob. & Ín. saz. & Prob. & Ín. saz. & Prob. & Ín. saz. & Prob. \\
\hline Janeiro & 5,99 & $\mathbf{0 , 0 7}$ & 5,05 & 0,30 & 6,24 & $\mathbf{0 , 0 0}$ & 6,18 & 0,67 & 12,33 & 0,12 & 3,12 & 0,50 \\
\hline Fevereiro & $-6,51$ & 0,16 & $-5,48$ & 0,43 & $-6,47$ & 0,02 & $-9,17$ & 0,65 & $-15,97$ & 0,15 & 1,89 & 0,77 \\
\hline Março & $-2,45$ & 0,60 & $-0,63$ & 0,93 & $-6,20$ & $\mathbf{0 , 0 3}$ & 12,32 & 0,55 & $-15,08$ & 0,17 & $-1,71$ & 0,80 \\
\hline Abril & $-6,41$ & 0,17 & $-7,62$ & 0,27 & $-7,41$ & $\mathbf{0 , 0 1}$ & 2,47 & 0,90 & $-13,75$ & 0,22 & $-7,06$ & 0,28 \\
\hline Maio & $-8,56$ & $\mathbf{0 , 0 7}$ & $-11,84$ & 0,09 & $-6,44$ & 0,02 & $-11,91$ & 0,56 & $-16,95$ & 0,13 & 2,20 & 0,74 \\
\hline Junho & $-8,53$ & $\mathbf{0 , 0 7}$ & $-4,06$ & 0,56 & $-6,49$ & 0,02 & $-6,06$ & 0,77 & $-21,78$ & 0,05 & $-10,27$ & 0,12 \\
\hline Julho & $-9,72$ & 0,04 & $-8,46$ & 0,22 & $-8,14$ & 0,00 & $-6,74$ & 0,74 & $-39,86$ & $\mathbf{0 , 0 0}$ & $-0,36$ & 0,95 \\
\hline Agosto & $-6,79$ & 0,15 & $-7,71$ & 0,27 & $-5,81$ & 0,04 & $-0,34$ & 0,99 & $-16,33$ & 0,14 & $-3,44$ & 0,60 \\
\hline Setembro & $-5,15$ & 0,27 & $-12,30$ & 0,08 & $-7,36$ & 0,01 & $-4,33$ & 0,83 & $-7,11$ & 0,52 & $-1,89$ & 0,77 \\
\hline Outubro & $-5,91$ & 0,20 & $-9,93$ & 0,15 & $-5,22$ & 0,06 & $-4,56$ & 0,82 & $-11,95$ & 0,28 & $-6,15$ & 0,35 \\
\hline Novembro & $-1,90$ & 0,69 & $-5,22$ & 0,45 & $-5,25$ & 0,06 & $-1,66$ & 0,94 & $-6,89$ & 0,53 & $-1,96$ & 0,77 \\
\hline Dezembro & $-8,28$ & 0,08 & $-7,28$ & 0,29 & $-6,91$ & 0,01 & $-22,39$ & 0,28 & $-6,66$ & 0,55 & $-1,17$ & 0,86 \\
\hline Estatística-F & 0,82 & 0,62 & 0,64 & 0,79 & 1,11 & 0,36 & 0,34 & 0,97 & 1,64 & 0,10 & 0,64 & 0,79 \\
\hline
\end{tabular}

Obs.: Valores em negrito indicam significância estatística a $10 \%$.

sentam-se amplamente diferenciados de acordo com o período de tempo utilizado como amostra na regressão.

Tomando todo o período, de janeiro de 1946 a dezembro de 2000, observou-se que apenas alguns índices sazonais (da equação 17 apresentada anteriormente) se apresentaram como estatisticamente significativos (valores em negrito).

O índice do mês-base (janeiro) e os índices dos meses de maio, junho, julho e dezembro, de maneira geral, confirmam os resultados discutidos na análise de sazonalidade pelos gráficos dos preços, isto é, os valores estimados indicam que o mês de janeiro se caracteriza como um mês de preços em elevação, com uma valorização média de 5,99 centavos de US\$ por libra-peso, enquanto os meses de maio, junho e julho correspondem a meses de desvalorização em relação às cotações observadas para janeiro. As desvalorizações médias nas cotações verificadas, relacionadas a esses meses, foram, respectivamente, de 8,56; 8,53; e 9,72 centavos de US\$ por libra-peso, em relação aos valores do mês-base de janeiro. Ao contrário do que era aparente nos gráficos, o mês de dezembro também se caracterizou como um mês de desvalorização da commodity para o período analisado como um todo.

Ao que se refere à natureza estocástica da sazonalidade, os resultados apresentaram duas características marcantes: primeiramente, indicaram que não há necessidade de se implementar o processo de diferenciação sazonal na série, pois não foram verificadas raízes unitárias sazonais para os preços do café. Em segundo lugar, pode-se verificar que esses preços tendem a ser correlacionados de maneira sazonal, mas que essa relação só é forte de um ano para o outro e não para períodos mais distantes no tempo.
Portanto, pode-se inferir, que de fato a sazonalidade nos preços do café brasileiro no mercado spot de Nova Iorque foi verificada a partir dos testes estatísticos efetuados. Todavia, deve-se ressaltar, que essa relação está se modificando ao longo do tempo, o que caracterizaria uma sazonalidade do tipo estocástica e não do tipo determinística para os preços desta commodity no mercado internacional.

Dados os resultados acerca da sazonalidade dos preços, tem-se que os produtores devem ter certa atenção no planejamento da venda de sua safra, no que tange aos meses mais favoráveis para essa venda. Verificou-se, que em média, os meses de junho a setembro se configuram como meses de safra no Brasil, e o nível dos preços da commodity, nesse período, tende a cair significativamente.

\section{Conclusões}

O mercado spot do café caracteriza-se como sendo marcado por acentuadas flutuações de preços. Essas flutuações implicam que a rentabilidade nessa atividade deverá oscilar de maneira significativa, o que pode propiciar, para os investidores mais informados, um diferencial de lucratividade, pois as suas decisões de hedge e de especulação estarão baseadas em previsões mais precisas acerca do comportamento do mercado.

No entanto, pode-se observar, que essas possibilidades de ganho ainda não são plenamente aproveitadas por grande parte dos agentes econômicos envolvidos nesses mercados, dada a imperfeita compreensão acerca da natureza das flutuações dos preços no mercado de café, que, se não podem ser evitadas, ao menos deveriam ser utilizadas pelos agentes econômicos ligados a esse 
mercado para otimizarem suas decisões e maximizarem seus lucros individuais.

Em termos gerais, pretendeu-se neste trabalho de pesquisa detectar e analisar a existência e a magnitude dos componentes estocásticos (e/ou determinísticos) de tendência, ciclos e sazonalidade na série temporal dos preços do mercado spot do café brasileiro na bolsa de Nova Iorque (NYBOT) para a segunda metade do século passado, mais especificamente, o período compreendido entre janeiro de 1946 a dezembro de 2000.

Optou-se pelo estudo do mercado de café, uma vez que esta é a principal commodity agropecuária do Brasil transacionada em bolsas de mercadorias e de futuros (aproximadamente 67\% dos contratos agropecuários transacionados na BM\&F são de café). Optou-se também pela análise dos preços no mercado internacional, pelo fato do Brasil ser o maior produtor e exportador mundial de café (30\% da produção mundial em média); dessa forma, trata-se de uma commodity cuja formação do preço deve contemplar principalmente o mercado internacional.

Os resultados encontrados mostraram que a tendência existente nos preços do café se configurou como uma composição de tendências dos tipos determinística e estocástica, ou seja, a série de preços do café brasileiro não se configurou como estacionária no período analisado.

Com relação aos ciclos existentes nos preços do café, tem-se que a análise espectral para os dados conjuntos de toda a amostra (janeiro de 1946 a dezembro de 2000) confirmou a incidência de um ciclo de média duração existente no intervalo de 22 a 44 meses. Subdividindo a análise por décadas, a fim de verificar mudanças na periodicidade dos ciclos, constatou-se a existência de um ciclo bienal (24 meses) como o mais recorrente, e que possi- velmente esteve ligado diretamente ao ciclo biológico do cafeeiro. Todavia, duas exceções ocorreram: verificouse que um ciclo de longo prazo nos preços do café, de aproximadamente 60 meses (cinco anos) de duração, também foi marcante na década de 60 e que na década de 90 pôde-se observar uma mudança no comportamento dos preços em termos do componente de ciclos. Nesta última década, o ciclo bienal não foi significativo, apenas um ciclo com freqüências correspondentes ao período de 12 meses, o que configuraria um ciclo sazonal.

Em relação à análise da sazonalidade nos preços, observou-se que para as décadas tomadas individualmente os resultados não diferiram significativamente dos verificados para todo o período. Observou-se, que a sazonalidade enquanto um componente de influência no comportamento dos preços do café no mercado internacional, não é da forma determinística e regular. Pelo contrário, ela é de natureza estocástica, variando em função do período do tempo.

Finalmente, deve-se citar que a presente pesquisa não está livre de limitações. Nesse sentido, sugere-se a realização de novos estudos sobre o mesmo tema. Poderiam ser utilizados diferentes períodos de tempo e diferentes metodologias para modelagem dos componentes das séries de preços, como a abordagem Bayesiana de West e Harrison (1989) para modelagem dos ciclos e da sazonalidade, ou a metodologia dos modelos markovianos para séries com mudança de regime, conforme apresentado em Brooks (2002). Sugere-se ainda, que análises bivariadas possam ser feitas analisando-se em conjunto a série spot dos preços e uma série com os preços futuros da commodity, para modelagem da interdependência desses preços.

\title{
Trend, cycles and seasonality in spot prices of brazilian coffee at the NYBOT
}

\begin{abstract}
The main aim of this research was to detect the existence of the stochastic (and/or deterministic) components of Trend, Cycle and Seasonality in the spot market prices of the most important Brazilian agricultural commodity: coffee. The methodology used was the analysis in the time domain (to trend and seasonality) and the analysis in the frequency domain, also known as spectral analysis for the study of cycles in prices. The results found that the trend in coffee prices is compounded by a mix of deterministic and stochastic trends. With relation to the cycles in the prices, the spectral analysis to whole sample data (from January 1946 to December 2000) has confirmed the rate of a cycle of medium duration between the interval of 22 and 44 months. With relation to seasonality, the research found that this component of the price series has had a very unstable characteristic throughout the decades analyzed. It has a stochastic nature and varies in function of time.
\end{abstract}

Keywords: Trend. Cycles. Seasonality. Coffee prices. 
ABRAHAM, B.; LEDOLTER, J. Statistical methods for forecasting. 1. ed. New York: John Wiley \& Sons, 1983. $445 \mathrm{p}$.

BOWERMAN, B. L.; O'CONNEL, R. T. Time series and forecasting: an applied approach. Massachusetts: California Duxbury Press, 1979. 481 p.

BOX, G. E. P.; JENKINS, G. M. Time series analysis: forecasting and control. Revised edition, San Francisco: Holden-Day Press, 1976. 575p.

BROOKS, C. Introductory econometrics for finance. 1. ed. Cambridge: Cambridge University Press, UK, 2002. 701 p.

CAMPBELl, J. Y.; LO, A. W.; MacKINLAY, A. C. The econometrics of financial markets. New Jersey: Princeton University Press, 1997. 611 p.

CHATFIELD, C. The analysis of time series - an introduction. 5. ed. London: Chapmam \& Hall, 1996. 286 p.

ENDERS, W. Applied econometric time series. 1. ed. New York: John Wiley \& Sons, 1995. 433 p.

HAMILTON, J. D. Time series analysis. 1. ed. New Jersey: Princeton University Press, 1994. 799 p.

HARVEY, A. Time series models. 1. ed. New York: John Wiley \& Sons Inc, 1981. 230 p.
HARVEY, A. Forecasting, structural time series models, and the Kalman filter. 1. ed. Cambridge: Cambridge Univesity Press, 1989. 554 p.

JUDGE, G. G. et al. Introduction, to the theory and pratice of econometrics. 2. ed. New York: John Wiley \& Sons, 1988.

PINDYCK, R. S.; RUBINFELD, D. L. Econometric models and economic forecasts. 4. ed. New York: Irwin/McGraw-Hill, 1998.

RAUSSER, G. C.; CARGILL, T. F. The existence of broiler cycles: an application of spectral analysis. American journal of agricultural economics, Oxford: Blackwell Publishing, v. 52, n. 1, p. 109-121, feb. 1970. Published as Journal of Farm Economics since 1919 to 1967.

RODRIGUES, R. O papel do setor privado e os novos desafios do abastecimento nacional. Revista de política agrícola, Brasília: Editora da CONAB, v. 10, n. 1, p. 1-15, jan-mar, 2001.

SOUZA, R. C. Modelos estruturais para previsão de séries temporais: abordagens clássica e bayesiana. 1. ed. Rio de Janeiro: Editora do Instituto de Matemática Pura e Aplicada (IMPA), 1989.

VANDAELE, W. Applied time series and Box-Jenkins models. 1. ed. New York: Academic Press, 1983. 417 p.

WEST, M.; HARRISON, J. Bayesian forecasting and dynamic models. 1. ed. New York: Springer-Verlag, 1989. 700 p.

\section{Sobre o autor}

\section{Wagner Moura Lamounier}

Economia Aplicada,UFV, Centro de Pós-graduação e Pesquisas em Contabilidade e Controladoria - CEPCON, Universidade Federal de Minas Gerais - UFMG,

Rua Curitiba, 832, sala 703, $7^{\circ}$ andar, Centro, CEP 30170120, Belo Horizonte, MG, Brasil,

e-mail: wagner@face.ufmg.br 
